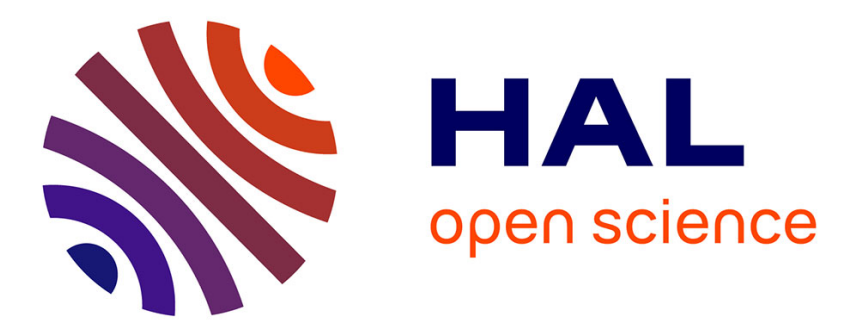

\title{
Les sédiments, documents historiques: lecture de la stratigraphie d'un habitat médiéval. Lac de Charavines, Colletières (Isère)
}

Jacques-Léopold Brochier

\section{- To cite this version:}

Jacques-Léopold Brochier. Les sédiments, documents historiques: lecture de la stratigraphie d'un habitat médiéval. Lac de Charavines, Colletières (Isère). Gallia - Fouilles et monuments archéologiques en France métropolitaine, 1986, 44 (1), pp.175-189. 10.3406/galia.1986.2860 . hal-01940952

\section{HAL Id: hal-01940952 \\ https://hal.science/hal-01940952}

Submitted on 27 Feb 2020

HAL is a multi-disciplinary open access archive for the deposit and dissemination of scientific research documents, whether they are published or not. The documents may come from teaching and research institutions in France or abroad, or from public or private research centers.
L'archive ouverte pluridisciplinaire $\mathbf{H A L}$, est destinée au dépôt et à la diffusion de documents scientifiques de niveau recherche, publiés ou non, émanant des établissements d'enseignement et de recherche français ou étrangers, des laboratoires publics ou privés.

\section{(ㅇ)(1) $\$$}

Distributed under a Creative Commons Attribution - NonCommercial - NoDerivatives| 4.0 


\title{
LES SÉDIMENTS, DOCUMENTS HISTORIQUES : LECTURE DE LA STRATIGRAPHIE D'UN HABITAT MÉDIÉVAL Lac de Charavines, Colletières (Isère)
}

\author{
par Jacques-Léopold BROCHIER
}

[Le lecteur sera peut-être étonné de l'étude d'un site qui ne rentre pas exactement dans le cadre chronologique de Gallia. C'est que cet article ne porte pas en fait sur l'histoire du site lui-même, mais sur une méthode consistant à considérer le sédiment comme un document et visant à une lecture stratigraphique la plus poussée possible. Cette démarche de recherche peut donc s'appliquer à n'importe quel site terrestre ou lacustre, et de n'importe quelle période. Or le sédiment est toujours le premier témoin dont le fouilleur dispose, en dehors même de tout mobilier archéologique. Il ne devrait jamais être détruit sans une étude scientifique préalable.

\section{La Rédaction]}

Depuis 1972 l'équipe du Centre d'Archéologie Historique des Musées de Grenoble et de l'Isère, dirigée par M. M. Colardelle, fouille un habitat médiéval du $x^{e}$ s., immergé sous les eaux du lac de Paladru à Charavines-les-Bains (Isère), à $40 \mathrm{~km}$ au n-o. de Grenoble. La conservation du matériel organique, des objets et structures architecturales en bois, due aux conditions sub-aquatiques, fait le grand intérêt de ce site situé à la charnière historique de l'an mille. Les premiers résultats de la fouille, conduite sous l'eau entre 1 et $2 \mathrm{~m}$ de profondeur, donnent l'image d'une société semi-seigneuriale, semi-paysanne'. Deux bâtiments, I et II, ont nettement été mis en évidence (fig. 1). La surface du

1 R. Colardelle et M. Colardell.e, L'habilat médiéval immergé de Colletières à Charavines (Isère), premier bilan des fouilles, dans Archéologie médiévale, X, 1980, pp. 167-269. bâtiment I peut être estimée de 200 à $240 \mathrm{~m}^{2}$, et la période d'occupation à une trentaine d'années ${ }^{2}$.

Une attention particulière a été portée aux études permettant la reconstitution des milieux naturels et des rapports que les habitants pouvaient entretenir avec ce dernier. Sous l'impulsion de M. Colardelle des études de macrorestes végétaux, de palynologie, de sédimentologie, de zoologie ont pû être réalisées. Plusieurs carottages recoupant les sédiments lacustres sus et sous-jacents à l'habitat ont été effectués sur le site, et en dehors du site habité, jusqu'à $-30 \mathrm{~m}$ de fond, pour reconnaître l'évolution des milieux $^{3}$.

De plus une étude axée uniquement sur les dépôts archéologiques d'origine anthropique a été entreprise. Ces dépôts sont ici très organiques et très bien conservés; ils se superposent sur un peu moins d'un mètre d'épaisseur correspondant à une occupa-

2 L. L.востьт, Elude dendrochronologique du batiment $I$. Annexe de op. cit. note 1, 1980. - L. Leboutet, M. Colardelle, E. Charles et B. Dangriacx, L'étude dendrochronologique de l'habilal médiéval immergé de Colletières, à Charavines (Isère): Interprélations archéologiques, dans Archéologie Médievvale, XIII, 1983, pp. 131-154.

3 J.-L. Borei, J. L. Brochier, K. Lundstrom-Baudais, M. Colardetle, Une expérience de recherche concertée sur le paléoenvironnement de l'habitat médiéval immergé de Colletières (Charavines, Isère): Sédimentologie, pollens, macrorestes végétaux, dans Palynologie archéologique, Notes et monographies techniques no 17 du CRA, CNRS, 1985. - J.-L. BoreL, J.-L. Brochite, K. Lundstrom-Baudais, Water level fluctualions of the lake Paladru (Isère, France), in the Xth and $X I t h$ centuries A.D., dans Paleohydrological changes in the temperate zone in the last 15.000 years, Ecologia Mediterranea, XI, 1985, pp. 179-183. 


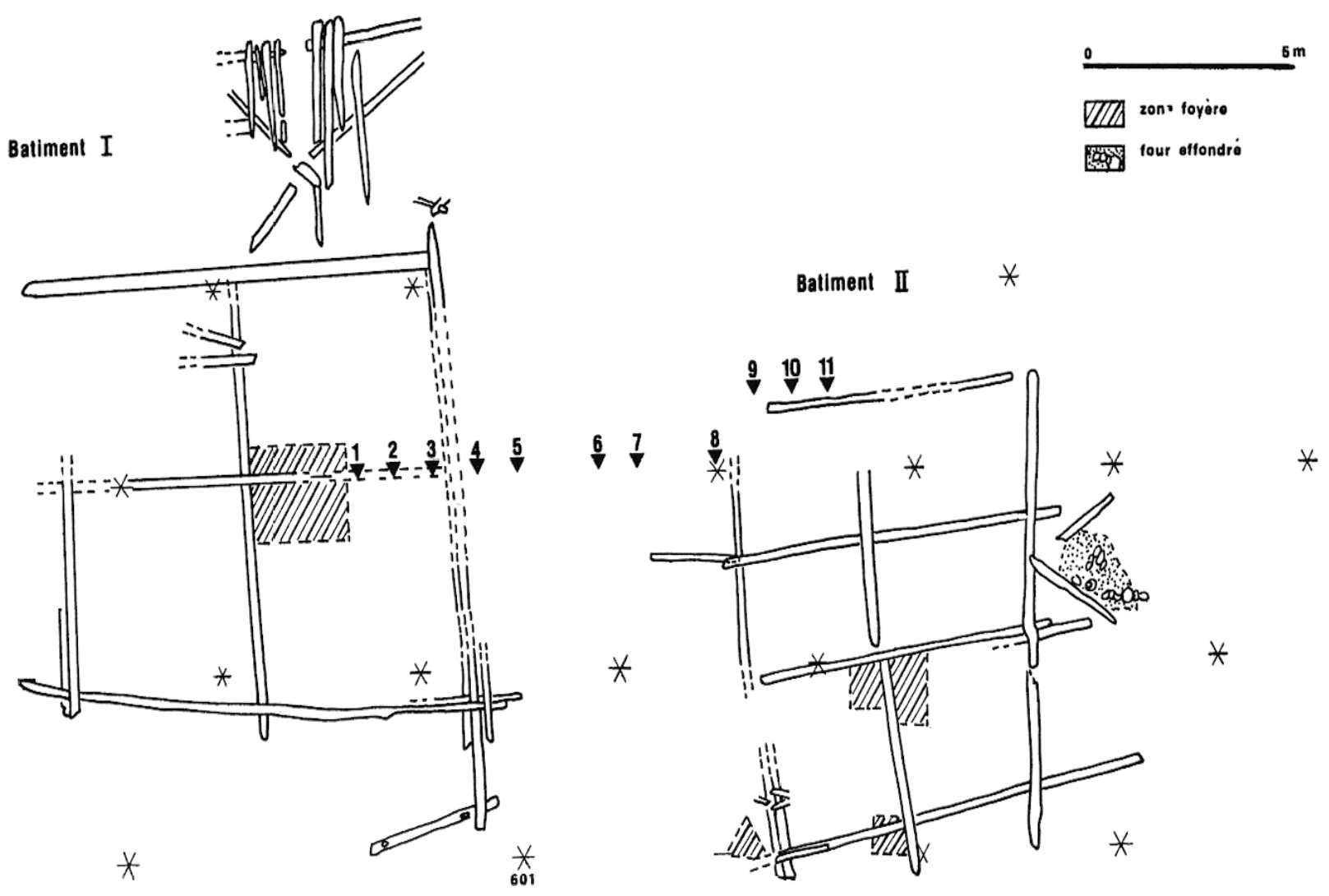

1 Charavines, station médiévale de Colletieres. Plan des bâtiments I et II, matérialisés par des madriers couchés empilés alternativement à angles droits. Répartition des carottes 1 à 11 .

tion de courte durée. La lecture d'une stratigraphie, à l'aide de l'étude sédimentologique, permet d'obtenir des données encore trop peu recherchées sur l'histoire d'un site. C'est cette méthode, appliquée à l'exemple de la séquence sédimentaire de l'habitat de Colletières que nous présentons ici.

$$
* *
$$

Les recherches se sont orientées sur trois axes :

1. Tout d'abord, le sédiment doit être considéré comme un document. - Le sédiment, de même que le mobilier archéologique qu'il contient, est une archive qui a enregistré à sa façon une partie de la vie des hommes que l'archéologue s'attache à restituer. La stratigraphie est la science de base de la fouille archéologique. On l'a considérée seule- ment comme la notation indispensable d'une succession de couches, correspondant à une notation de provenance sur chaque objet. Ces couches ne sont pas qu'un simple emballage de vestiges archéologiques; leur organisation, leur composition, leur faciès sédimentaire sont justement le reflet de la vie des hommes, de leurs activités, de leur histoire sur le sile que l'on étudie, ainsi que de l'emprise du milieu naturel. Ces informations il faut apprendre à les lire dans le sédiment. On parle souvent « d'archives du sol "; le sol lui même, en premier, est une archive en l'absence même de tout document archéologique.

2. Le sédimenl est un documenl elhnographique et historique. - Les caractères sédimentaires de texture, structure, couleur, composition, tels qu'ils sont déterminés au chapitre suivant, permettent de définir des faciès sédimentaires, de les suivre, 


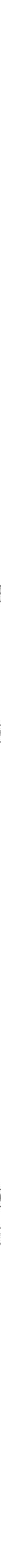


de les cartographier. Ces faciès, s'ils ne sont pas d'origine naturelle sont à rattacher aux activités de l'homme. Construction, destruction, emploi de matériaux divers, stabulation, parcage, déplacements, utilisation du feu, rejets de déchets, etc., engendrent des sédiments spécifiques, ou marquent le sédiment de façon caractéristique. Les variations de la nature des sédiments à la surface d'une même occupation, l'anthropisation des dépôts, vont donc nous permettre de mieux comprendre l'organisation de l'habitat et la vie des habitants ${ }^{4}$. En passant d'une lecture "horizontale " à une lecture "verticale", les sédiments se superposent, enregistrent le temps et les divers événements qui ont marqué l'histoire de l'implantation humaine. Mais cet enregistrement n'est, pas forcément, continu : la séquence, sédimentaire, peut être entrecoupée de lacunes, des sédiments différents trouvés au même niveau n'étant pas forcément synchrones.

3. L'habitat médiéval de Colletières, étude-test d'une séquence archéologique. - L'intérêt de cette succession de niveaux est sa conservation et sa dilatation; on trouve là 30 à $70 \mathrm{~cm}$ de dépôts, présentant des natures et des structures variées pour une période d'occupation courte, proche de trente années. La conservation du matériel organique en milieu sub-aquatique est bien sûr responsable de la qualité de l'enregistrement, mais on ne doit pas douter que, même en milieu terrestre, les sédiments peuvent être aussi de remarquables archives.

Nous avons d'abord cherché à construire un modèle, en utilisant et en nous aidant de structures d'habitats déjà reconnues, les deux bâtiments I et II, séparés par un espace ; d'où le choix d'un profil stratigraphique de $13 \mathrm{~m}$ de long, restitué d'après 11 carottes effectuées sur une ligne recoupant ces structures (fig. 1 et 2 ).

Un minimum d'analyses bien situées, servent à déterminer les corrélations chronostratigraphiques, à spécifier divers faciès sédimentaires, et à proposer une première interprétation sur les événements qui en sont à l'origine. Cette approche apporte des éléments destinés à la restitution historique,

4 J.-L. BrochIER, La sédimentologie lacusire spatiale: étude d'une surface d'habitat sur la station des Baigneurs, lac de Charavines, dans Bulletin de la Société préhistorique française, 79, 1982, no 2, pp. 51-55. - Id., L'habital lacustre préhistorique: problèmes géologiques, dans Archives des Sciences, Genève, 36, 1983, fasc. 2, pp. 247-260. - Id., L'habitat lacustre: les fumiers, couches archéologiques riches en matériel organique des stations littorales de la baie d'Auvernier, lac de Neuchatel, CH. Caractères sédimentologiques et processus de sédimentation. Manusc. $34 \mathrm{p}$. Musée Cantonal d'Archéologie, Neuchâtel, 1984, nº 36. éléments à vérifier, à compléter par les études archéologiques classiques ou plus spécialisées, tout ne s'inscrivant pas dans le sédiment. Des données essentielles sont notamment à attendre de l'étude de K. Lundstrom-BAudais sur les macrorestes végétaux qui constituent ici le matériel principal composant les dépôts ${ }^{5}$. L'extension des divers faciès, ainsi individualisés, sera relevée en planimétrie et sur profils verticaux. Ces premiers résultats serviront au cours de la fouille à l'enregistrement du mobilier archéologique, puis, lors de la réalisation des plans de répartition, où l'on court toujours le risque de superposer et de confondre plusieurs couches d'âges différents.

Il n'est pas possible dans ce type de sédimentation de définir des sols d'habitat, au sens d'une surface sur laquelle les hommes auraient marché, vécu; un même moment est représenté par une épaisseur de sédiment. Nous nous sommes surtout attachés à cerner les problèmes de synchronisme et diachronisme dans la succession des dépôts.

Sur le site de Colletières, une même occupation, d'une trentaine d'années seulement, est représentée par plusieurs couches de natures différentes. Les coupures sédimentaires ne correspondent pas systématiquement à des coupures culturelles, selon un principe que l'on a généralement tendance à appliquer. La conservation, ici, de la matière organique (végétaux, bois, fumiers) nous oblige à envisager le rôle indéniable que cette dernière a pu jouer sur les sites terrestres où elle ne s'est pas conservée, et lors de sa disparition, en ce qui concerne l'enregistrement stratigraphique. Cette étude montre encore que la fonction d'un bâtiment, par exemple, n'est pas fixée pour une même période, mais qu'elle peut évoluer. Il faut éviter de généraliser dans le temps une fonction reconnue à partir d'une seule observation archéologique clé (vestige particulier, structure), alors que le reste de la stratigraphie n'a pas encore été vraiment interprété.

Ainsi, l'étude sédimentologique approfondie d'un profil test, peut être considérée en un premier temps comme un outil de travail, un guide, une référence pour la poursuite des fouilles ; elle apporte des résultats appréciables, qui, avec l'ensemble des autres données, pourront servir à la restitution finale de l'histoire du village médiéval de Colletières.

\section{L'ÉTUDE SÉDIMENTOLOGIQUE.}

\section{TERRAIN, EGHANTILLONNAGE.}

Onze tubes de PVC, de $9 \mathrm{~cm}$ de diamètre et de $1 \mathrm{~m}$ de long, ont été enfoncés tous les mètres sur la ligne des triangles 202-201-203; les carottes 9, 10, 11, l'ont été sur une parallèle à cette ligne $2 \mathrm{~m}$ plus à l'est (fig. 1). Cette ligne

5 K. Lundstrom-Baudais, Etude préliminaire des macrorestes végétaux. Annexe IV, op. cit. note 1. 

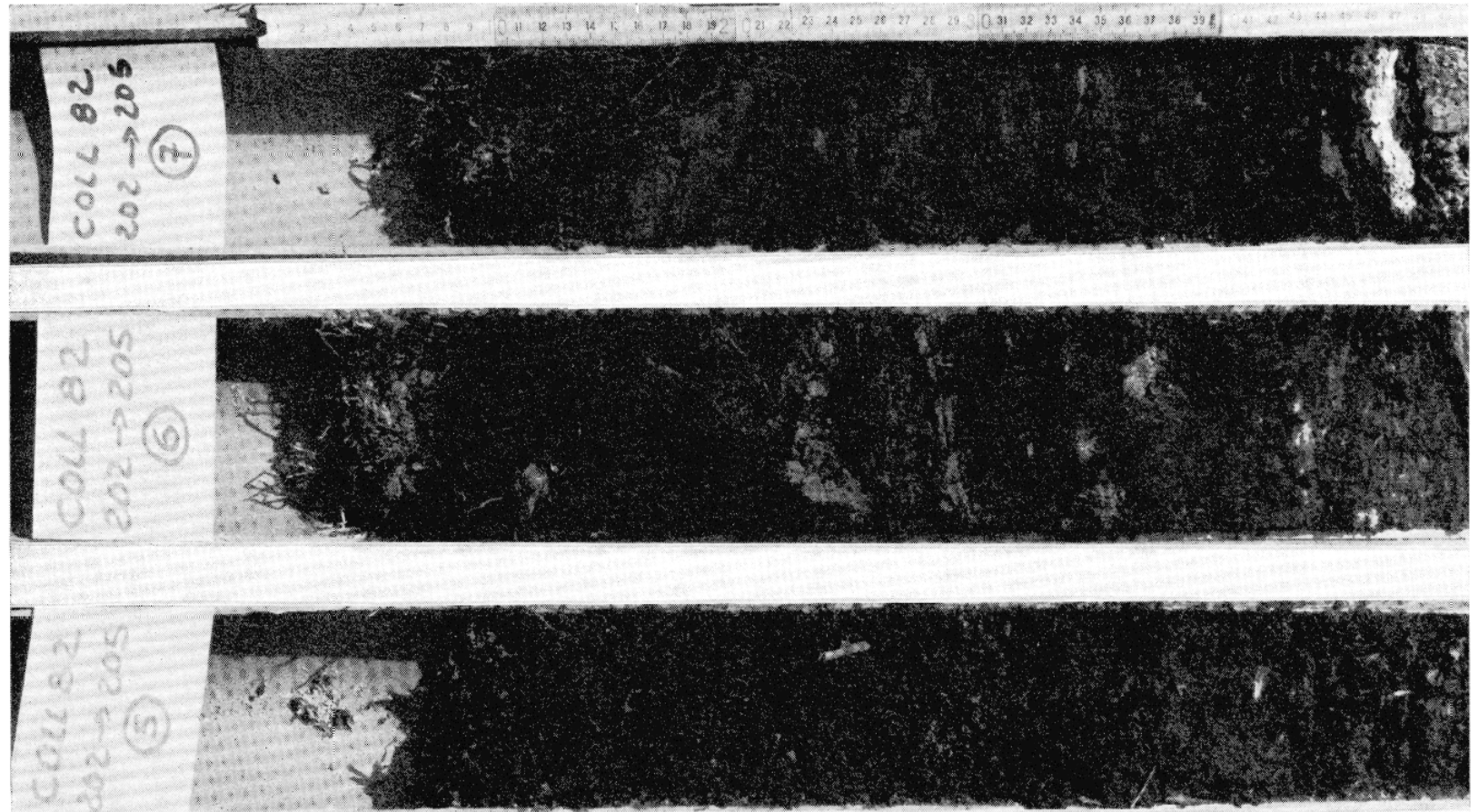

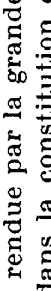
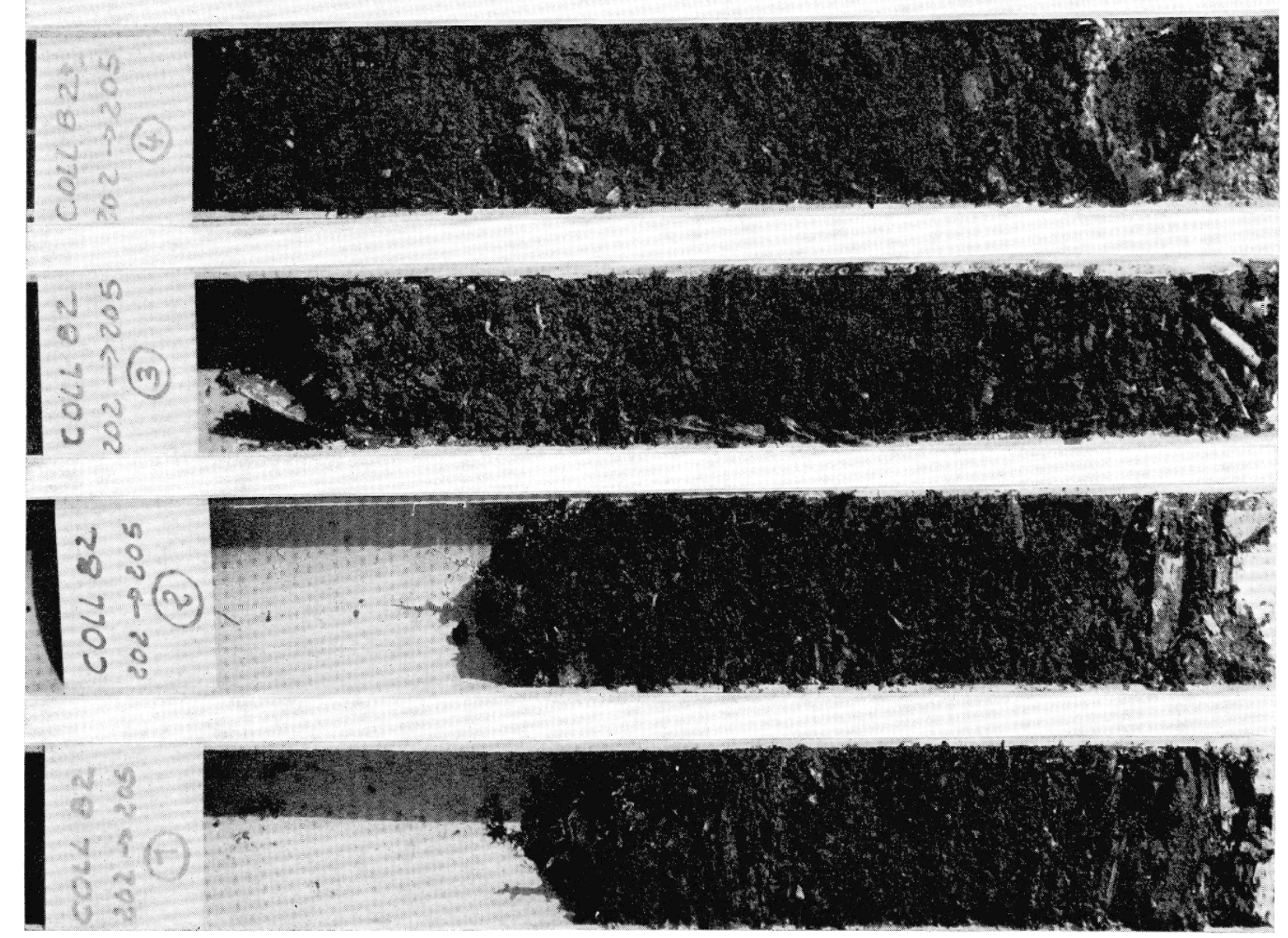
recoupe des structures déjà connues : bâtiment I, espace, bâtiment II et son auvent. Chaque carotte retirée est décrite, dessinée, photographiée permettant nombre d'observations qui ne sont pas possibles sous l'eau.

Les sédiments sont caractérisés en observation directe par leur structure, leur couleur, leur texture, leur composition (fig. 2). La structure, c'est l'organisation des éléments qui les composent ; elle peut être compacte, tassée, ou au contraire ouverte, laissant un espace entre les divers éléments, ou encore en particules isolées, ou en grumeaux. La texture, c'est la qualité granulométrique, argilcuse, limoneuse, sableuse, sablo-limoneuse, etc., selon l'importance de chaque classe de taille des grains. La qualité et la quantité des éléments qui constituent le sédiment sont appréciées au toucher et à la vue, parfois avec l'aide d'un microscope stéréoscopique.

L'échantillonnage est opéré en vue des analyses sédimentologiques (numérotation S, fig. 2), et de l'étude des macrorestes végétaux (numérotation $\mathrm{K}$, fig. 2). Un minimum de $200 \mathrm{~g}$ de sédiment est prélevé pour chaque discipline, à chaque variation des dépòts le long d'une carotte. Le diamètre des tubes n'a pas toujours permis de réaliser un échantillonnage complet et jumelé sur toutes les carottes; on s'est attaché à obtenir des échantillons reflétant l'ensemble des variations de faciès.

\section{LABORATOIRE, ANALYSES.}

Texture et composition font l'objet d'analyses permettant d'obtenir des paramètres quantifiables. Nous ne décrirons pas ici les processus d'analyses adaptés aux sédiments des sites de bord de lac ${ }^{6}$. Nous rappellerons brievement les paramètres utilisés :

Paramètres concernant la texlure du sédiment (granulométrie définie par tamisage) : - pourcentage en sables grossiers de 2 à $0,6 \mathrm{~mm}$; - pourcentage en sables moyens de 0,6 à $0,2 \mathrm{~mm}$; - pourcentage en sables fins de 0,2 à $0,06 \mathrm{~mm}$; - pourcentage en limons + argiles, particules inférieures à $0,06 \mathrm{~mm}$.

Le terme de sable recouvre ici l'ensemble des particules, minérales et végêtales, de 0,06 à $2 \mathrm{~mm}$. Les courbes granulométriques cumulatives classiques n'ont pas été utilisées ici, dans ces sédiments très anthropisés.

Paramètres concernant la composition chimique (définis sur le sédiment inférieur à $0,5 \mathrm{~mm}$ ): - teneur en matière organique (liée à l'anthropisation); - teneur en matière organique très fine, notée "humus"; ne doit pas être prise au sens strict pédologique ; - teneur en phosphates (licé à l'anthropisation, aux déchets organiques divers); - teneur en carbonates (liée au lac, aux inondations); - le $\mathrm{pH}$ (valeurs plus basses, plus "acides", en présence de matière organique; valeurs plus fortes, plus "basiques", en présence de lessivages ou d'actions du lac).

Paramètres concernant la composition de la fraction sableuse. Le comptage des grains de la fraction de 0,6 à $2 \mathrm{~mm}$, observée

6 J.-L. Brochier, M. Joos, Un élément important du cadre de vie des néolithiques d'Auvernier-Port: le lac. Approche sédimentologique, dans A. Billamboz : La station néolithique d'Auvernier-Port. Cadre et évolution, dans Cahiers d'Archéologie Romande, 1982, Auvernier no 5, Lausanne, pp. 43-67. au stéréomicroscope, donne : - le pourcentage en débris végétaux (fragments de brindilles, feuilles, graines, etc.); - le pourcentage en débris minéraux (grains provenant de roches diverses:; - le pourcentage en éléments limniques (débris d'organismes vivant dans le lac : tests de mollusques aquatiques, petits arthropodes à valves tels que les ostracodes, ou encore oogones de characées et petites concrétions carbonatées d'origine aquatique dues à des bactéries).

Les apports détritiques sont ici d'origine essentiellement anthropique. Les débris végetaux sont toujours les plus abondants, amenés par l'homme pour son habitat, sa nourriture, ou celle des bêtes. Les débris minéraux sont plus rares; s'ils peuvent être dus à l'érosion des rives, ils proviennent surtout d'apports par l'homme pour ses constructions, et d'apports involontaires au cours d'activités agricoles. Les apports limniques peuvent indiquer des changements de niveau de l'eau ou parfois des apports anthropiques liés à des activités orientées vers le lac.

L'observation morphoscopique de cette fraction des sables a aussi permis, dans certains cas, d'établir la pétrographie des éléments minéraux pour préciser leur origine, de compter (en \% de l'ensemble des éléments) les charbons de bois afin de préciser les activités liées aux foyers, ou encore des agglomérats de matière organique fine que nous avons reconnu être du fumier d'étable ou de parcage.

\section{REPRESENTATION GRAPHIQUE, VA- RIATIONS LATÉRALES, CORRELATIONS CHRONOST RATIGRA PHIQUES.}

Les courbes de représentation classiques en logs (histogrammes suivant la stratigraphie) des valeurs des 15 paramètres sédimentologiques déterminés sur chacune des 11 colonnes ne permettent pas une comparaison spatiale efficace. Nous avons opté pour des diagrammes où chaque paramètre est étudié dans sa variation stratigraphique et latérale le long de la ligne de carottages.

Les diagrammes latéro-verticaux (fig. $4 \mathrm{a}, \mathrm{b}, \mathrm{c}, \mathrm{d}$ ). Ces diagrammes portent en abscisse les numéros de caroltes, et la valeur du paramètre en ordonnée; les échantillons ne sont plus dans leur ordre stratigraphique et sont repérés par leurs numéros. Comportant de nombreux échantillons de couches différentes, ils sont difficiles à lire mais néanmoins indispensables; ils permettent d'individualiser les couches par des teneurs plus ou moins fortes en un ou plusieurs éléments. Des valeurs-seuils sont déterminées (délimitées sur les diagrammes par la bande grisée), isolant ainsi un ensemble d'échantillons ayant le même caractère : échantillons dont la teneur en phosphates est supérieure à 7 unités couleur, en matière organique supérieure à $50 \%$, en débris minéraux supérieure à $20 \%$ de l'ensemble des éléments composant la fraction sableuse de 0,6 à $2 \mathrm{~mm}$, ou comprise entre 10 et $20 \%$, etc.

Les schémas straligraphiques (fig. 5 à 9 ). Ils permettent une visualisation spatiale des résultats obtenus sur les diagrammes latéro-verticaux. Les échantillons regroupés par les mêmes caractères sédimentaires, sont reportés graphiquement 

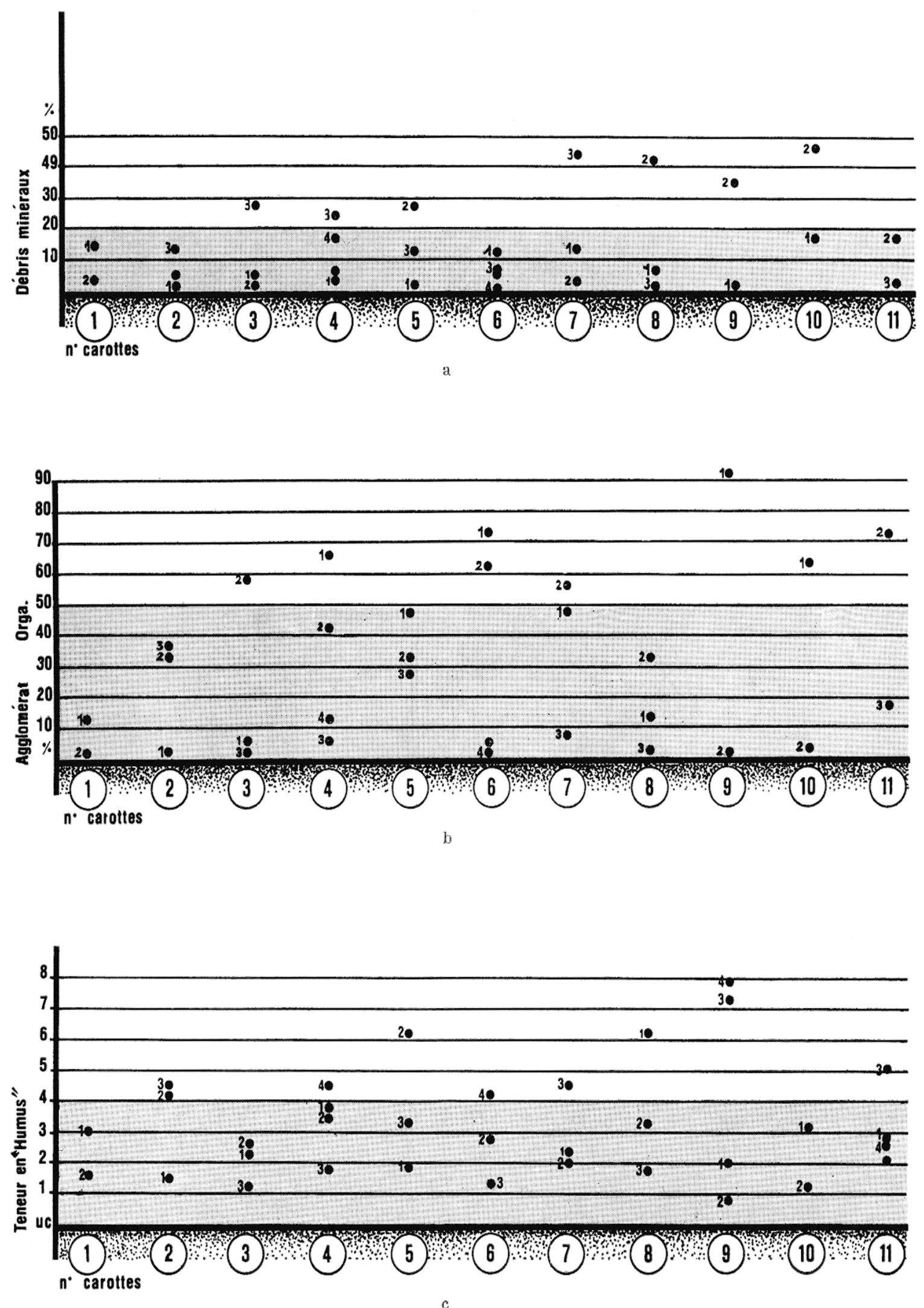


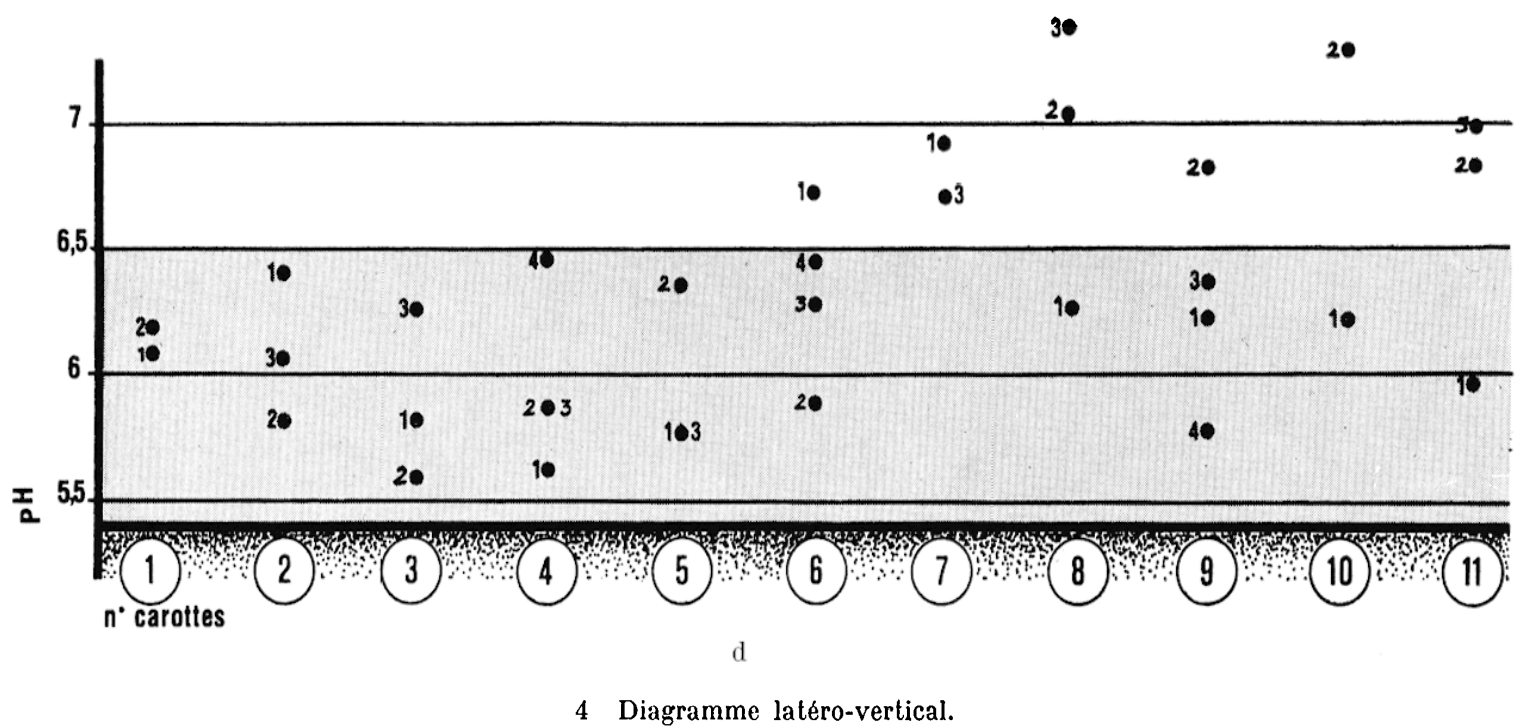

a. Individualisation et situation des échantillons (marqués $\mathrm{S}$, fig. 2) les plus riches en débris minéraux; teneurs supérieures à $20 \%$ (cf. fig. 5 ).

b. Individualisation et situation des échantillons (marqués S, fig. 2) les plus riches en agglomérats organiques (fumier) ; teneurs supérieures à $50 \%$ (cf. fig. 6).

c. Individualisation et situation des échantillons (marqués S, fig. 2) les plus riches en matière organique fine, "humus" . teneurs supérieures à 4 u.c. (unité couleur) (cf. fig. 5).

d. Individualisation et situation des échantillons (marqués S, fig. 2) les moins acides; pH supérieur à 6,5 (cf. fig. 7);

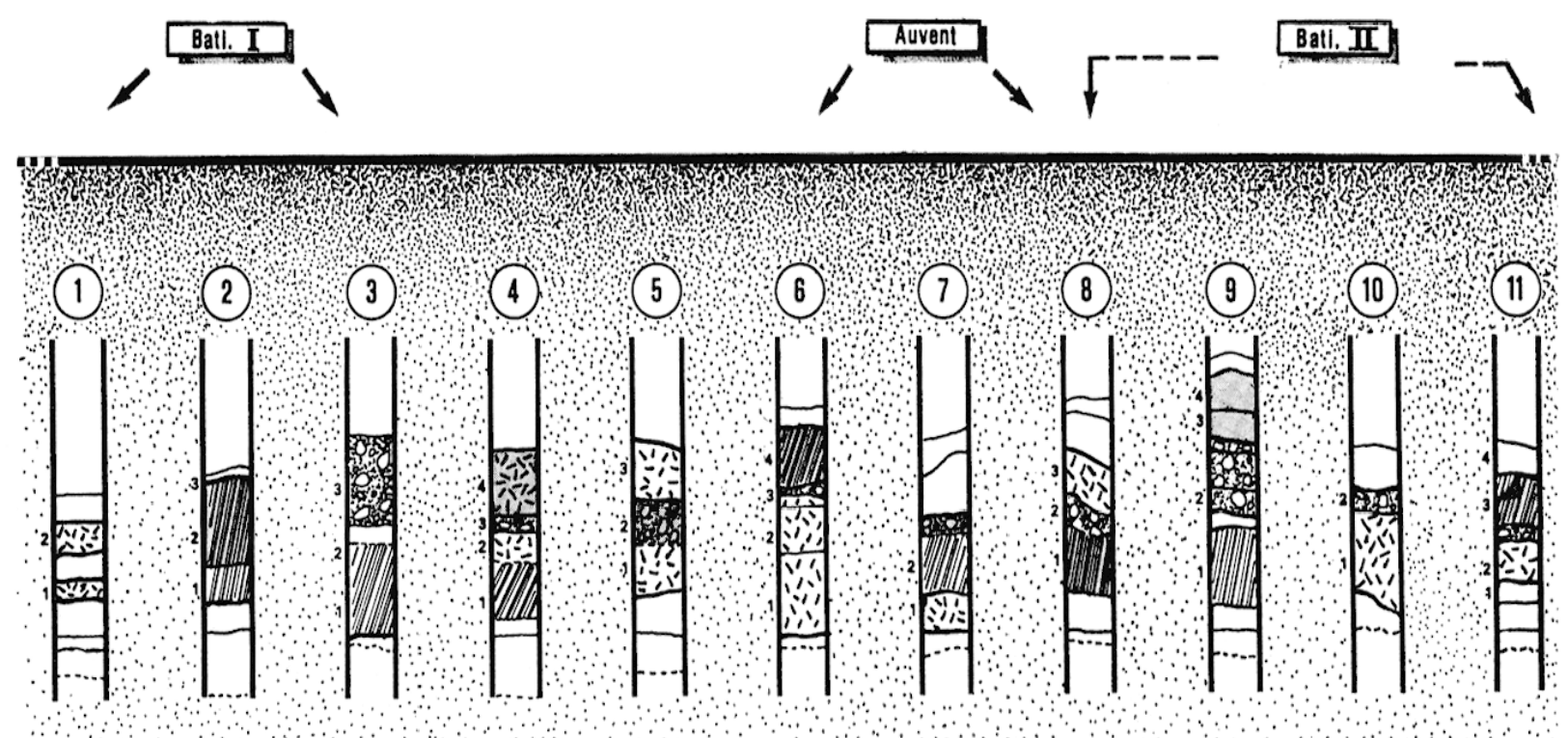

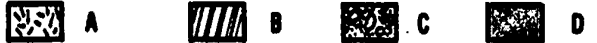

5 A, Niveaux dont la teneur en débris végétaux est supérieure à $80 \%$; B, niveaux dont la teneur en matière organique est supérieure à $50 \%$; C, niveaux dont la teneur en débris minéraux est supérieure à $20 \%$; Diveaux dont la teneur en matière organique fine, "humus", est supérieure à 4 u.c. (unité couleur). 

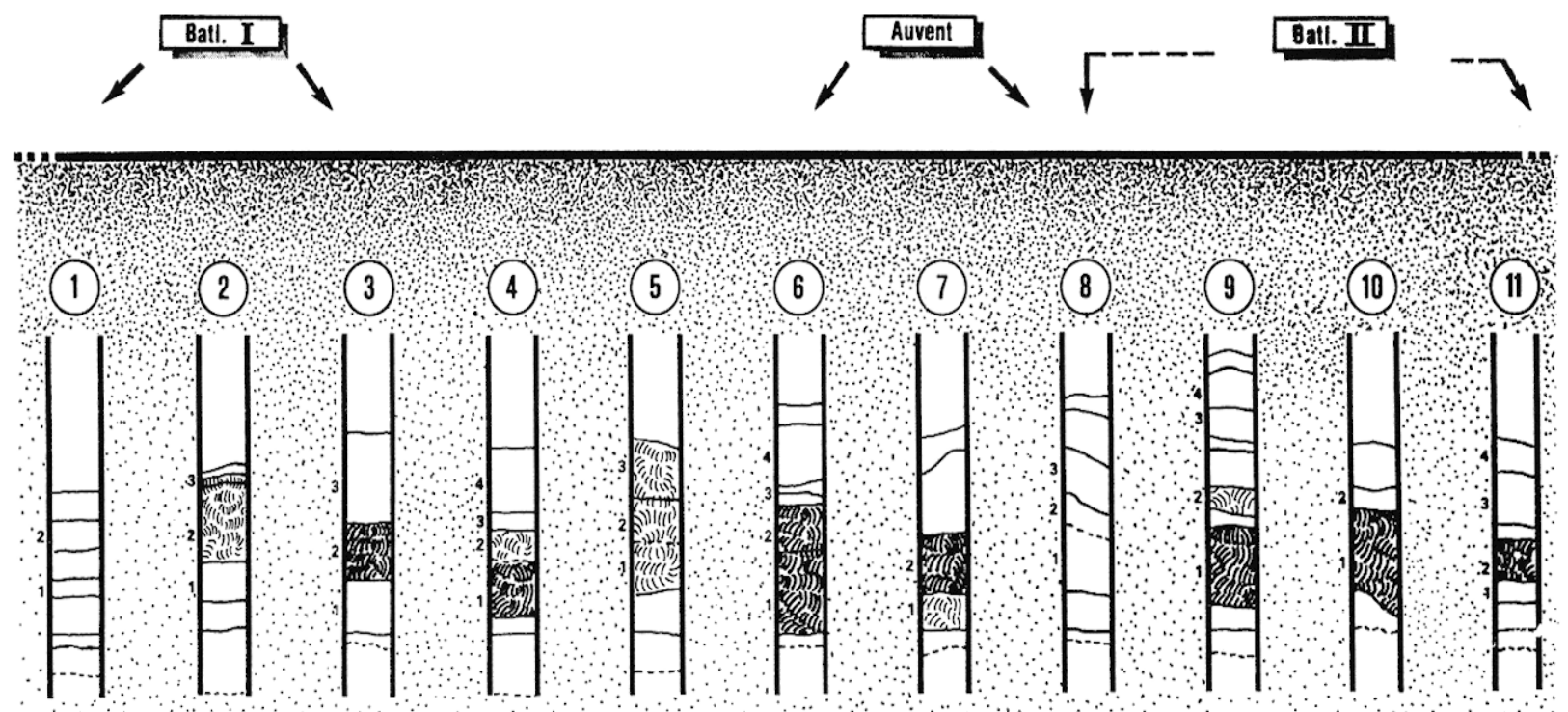

6 Niveaux dont la teneur en agglomérats organiques (fumier animal) est supéricure à $50 \%$ (figuré foncé), compris entre 20 et $50 \%$ (figuré clair).

7 Niveaux dont le $\mathrm{pH}$ est supérieur à 6,5 .
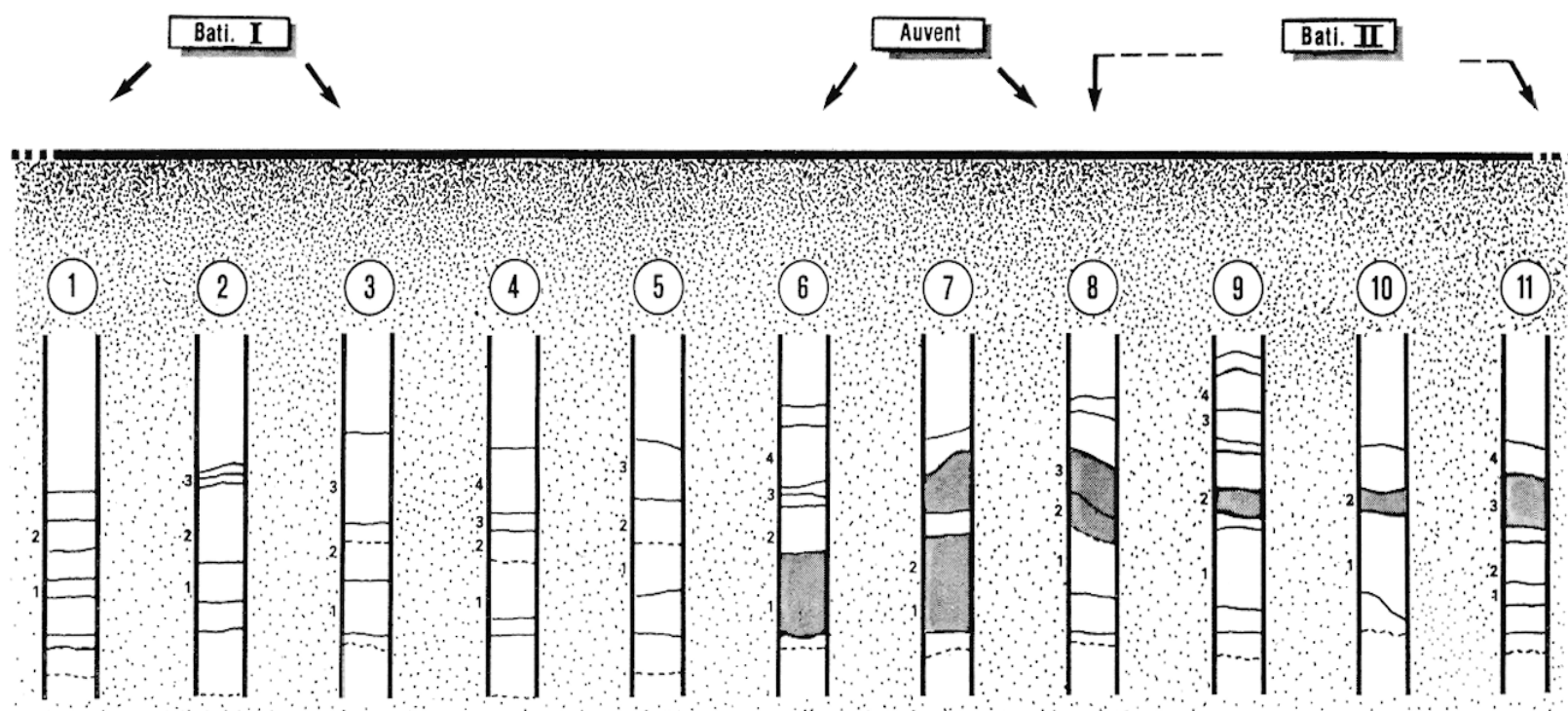


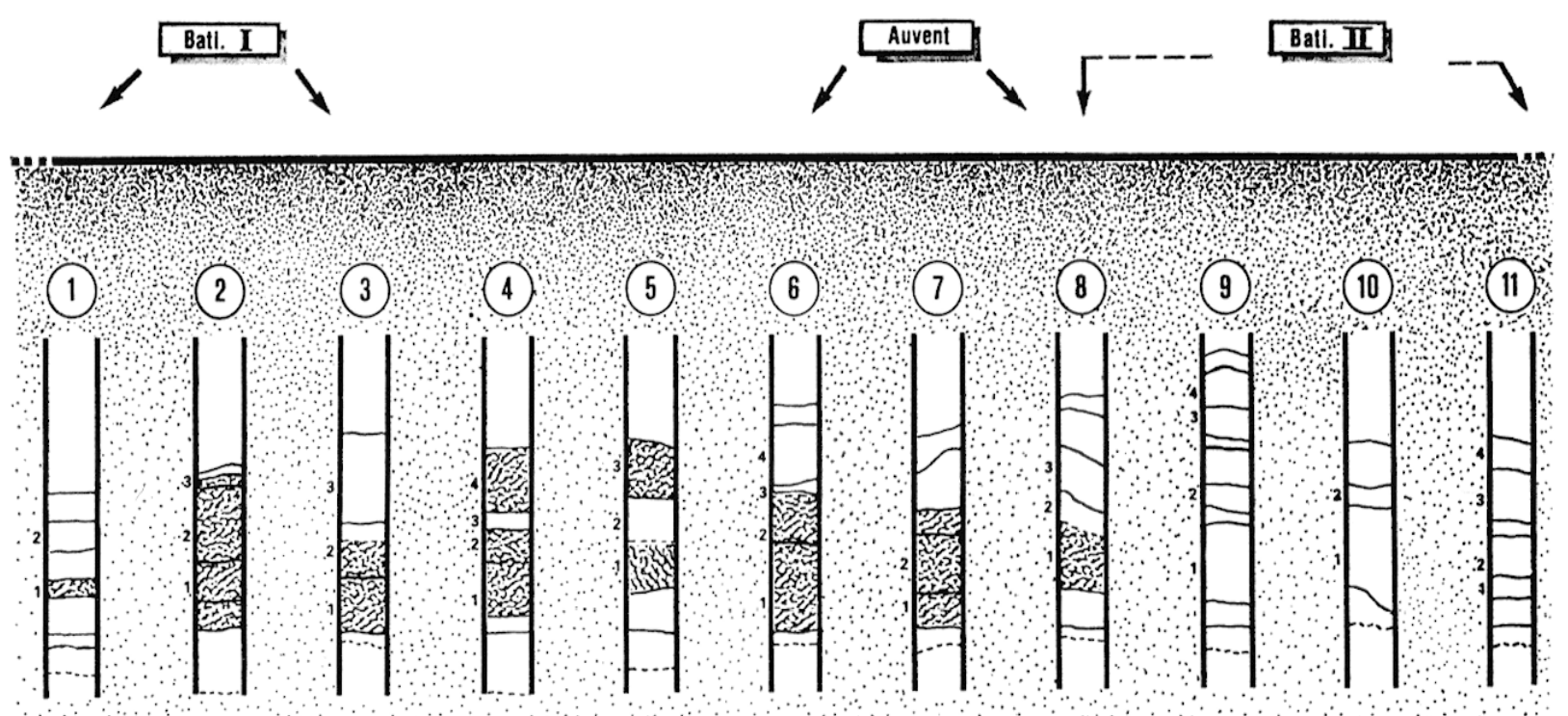

8 Niveaux dont la teneur en phosphates est supérieure à 7 unités couleur.

9 Niveaux dont la teneur en charbons de bois est supérieure à $50 \%$ (hachures foncées), comprise entre 20 et $50 \%$ (hachures claires).
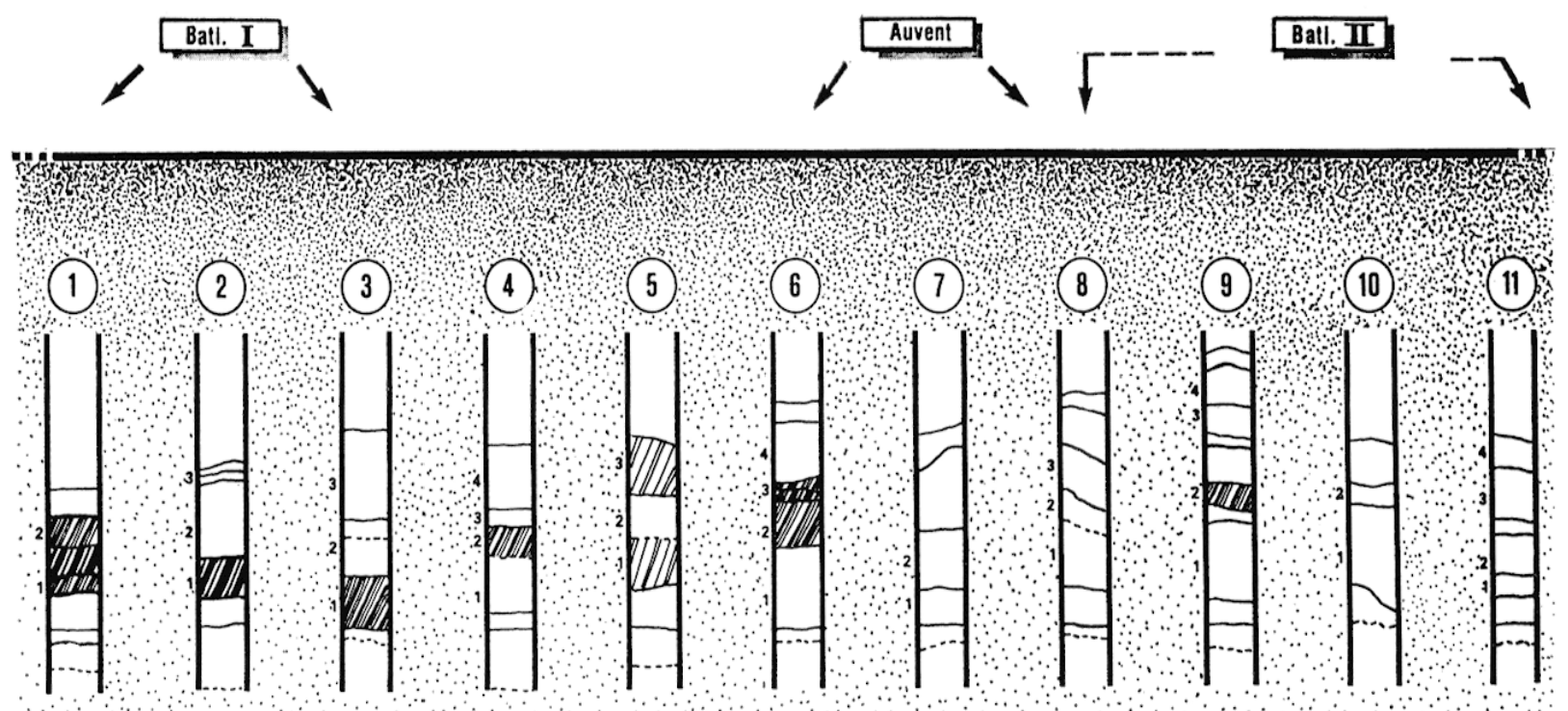
à leur place dans le schéma d'ensemble des carottes. On peut alors apprécier directement la répartition spatiale et temporelle des couches possédant les mêmes caractères. Il faut préciser que l'homogénéité apparente de la séquence sédimentaire (fig. 3) ne permet pas sur le terrain de faire toutes les distinctions stratigraphiques nécessaires.

La figure 5 montre nettement la place des niveaux riches en matière organique, séparés par un niveau plus riche en débris minéraux. La figure 6 montre que c'est le niveau inféricur qui est composé de fumier animal (agglomérats organiques). Les figures 7 et 8 mettent en évidence le fait que les couches dont le $\mathrm{pH}$ est supérieur à 7 sont liées seulement au bâtiment II et que les teneurs plus fortes en phosphates sont liées seulement au bâtiment I et à l'espace entre les deux maisons.

Ces schémas stratigraphiques nous ont permis de résoudre les problèmes de synchronisme et diachronisme. Nous avons considéré que sur un même niveau, un même faciès sédimentaire représentait une même ligne-temps. Nous n'avons pas la possibilité de déceler des diachronismes de faible amplitude; il est vrai qu'un même sédiment, à un même niveau, en des lieux différents, ne s'est pas forcément déposé dans le même temps. Sur cette période de trente ans, où six phases ont pu être définies, nous considérons ces corrélations comme représentant la même ligne-temps.

$$
\text { ** }
$$

Six phases sont proposées pour la constitution de la séquence sédimentaire étudiée; une phase scindant la vie du village en deux est individualisée (phase IV). Les analyses sédimentologiques n'ont à aucun moment permis de déceler la présence certaine d'eau au cours du dépôt successif des couches. Nous pouvons donc considérer que les habitations étaient construites sur terre ferme, en bordure du lac, ce qui concorde avec les observations faites par ailleurs sur la fouille archéologique. Des inondations très rapides ne sont pas exclues.

\section{PROPOSITIONS SUR LA RESTITUTION DES ÉVÉNEMENTS AYANT MARQUÉ LA VIE DES BÂTIMENTS I ET II, D'APRES LES RÉSULTATS DE CETTE ÉTUDE.}

\section{PHASE I (fig. 10).}

Cette phase donne lieu à une sédimentation de débris organiques grossiers, copeaux, brindilles, gros bois. La structure du dépôt est ouverte, non compactée. Les teneurs en matière organique el phosphates sont élevées (carottes 3 S1 et
$7 \mathrm{Sl}$ (fig. $4 \mathrm{c}, 58)$ ). Les charbons de bois sont déjà nombreux en $3 \mathrm{SI}^{7}$ (fig. 9). Aucune trace d'indices limniques et de carbonates n'est présente. On peut rattacher à cet épisode, de par leur structure, les couches de base de toutes les carottes, exceptées les 6 et 10. Noter l'absence de ce type de sédiment en $6 \mathrm{Sl}$, et probablement un remaniement avec la phase suivante dans la carotte 10 , où, dès la base, on trouve des traces de fumier animal.

En ll Sl une activité est génératrice de débris organiques fins.

Sans l'étudier ici, car ne faisant pas partie de la séquence complètement anthropiséc, nous signalerons seulement l'existence diune couche de charbons de bois flottés dans la craie sous-jacente se rattachant à une occupation non connue immédiatement antérieure (datages $14 \mathrm{C}$ pénécontemporains).

C'est la période de construction des maisons; construction puis habitation se succèdent rapidement. $\mathrm{La}$ régularité de ce niveau reposant sur la craic lacustre, plaide en faveur de la contemporanéité des constructions des deux bâtiments I et II. L'analyse des macrorestes végétaux apportera des données déterminantes sur ces débris. Le foyer du bâtiment I fonctionne très tôt (charbons de $3 \mathrm{~S} 1$ ). L'activité génératrice de phosphates, probablement domestique, que l'on suit tout au cours de l'existence du bâtiment I, est déjà décelable dès cette phase. La quasi inexistence de matériel minéral exclut des constructions où le pisé ait été largement employé; même l'utilisation du torchis parait devoir être mise en doute.

\section{PHASE II (fig. 11).}

Les carottes 3 à 11 , exceptée 8 , présentent toutes un sédiment très organique contenant un grand nombre d'agglomérats organiques (fig. $4 \mathrm{~b}$ et 6 ). Ces agglomérats, à matrice de matière organique fine d'aspect goudronneux, sont, comme nous avons pu le constater par comparaison avec des restes actuels, des éléments de fumier d'étable desséché, évolué et démantelé. Nous avons déjà décrit des formations identiques sur les sites préhistoriques d'Auvernier-Brise Lames, Auvernier-Nord et de Charavines-Baigneurs ${ }^{8}$; elles se trouvent sur des sites peu touchés par l'eau.

Les carottes 6, 7 et 9 montrent sur des épaisseurs de 10 à $20 \mathrm{~cm}$, une texture fine et une structure tassée d'aspect tout à fait identique à certains fumiers actuels.

Les dépôts de cette phase sont riches en phosphates pour les carottes 1 à 7 seulement, et ne concernent donc que le bâtiment I (fig. 8). Le pH est supérieur à 6,5 dans les carottes 6 et 7 (fig. 7).

$73 \mathrm{~S} 1=$ carotte $\mathrm{n}^{\circ} 3$, échantillon $\mathrm{S} 1: \mathrm{S}=$ sédimentologie, no l, cf. fig. 2.

8 Cf. note 4. 
Phase I
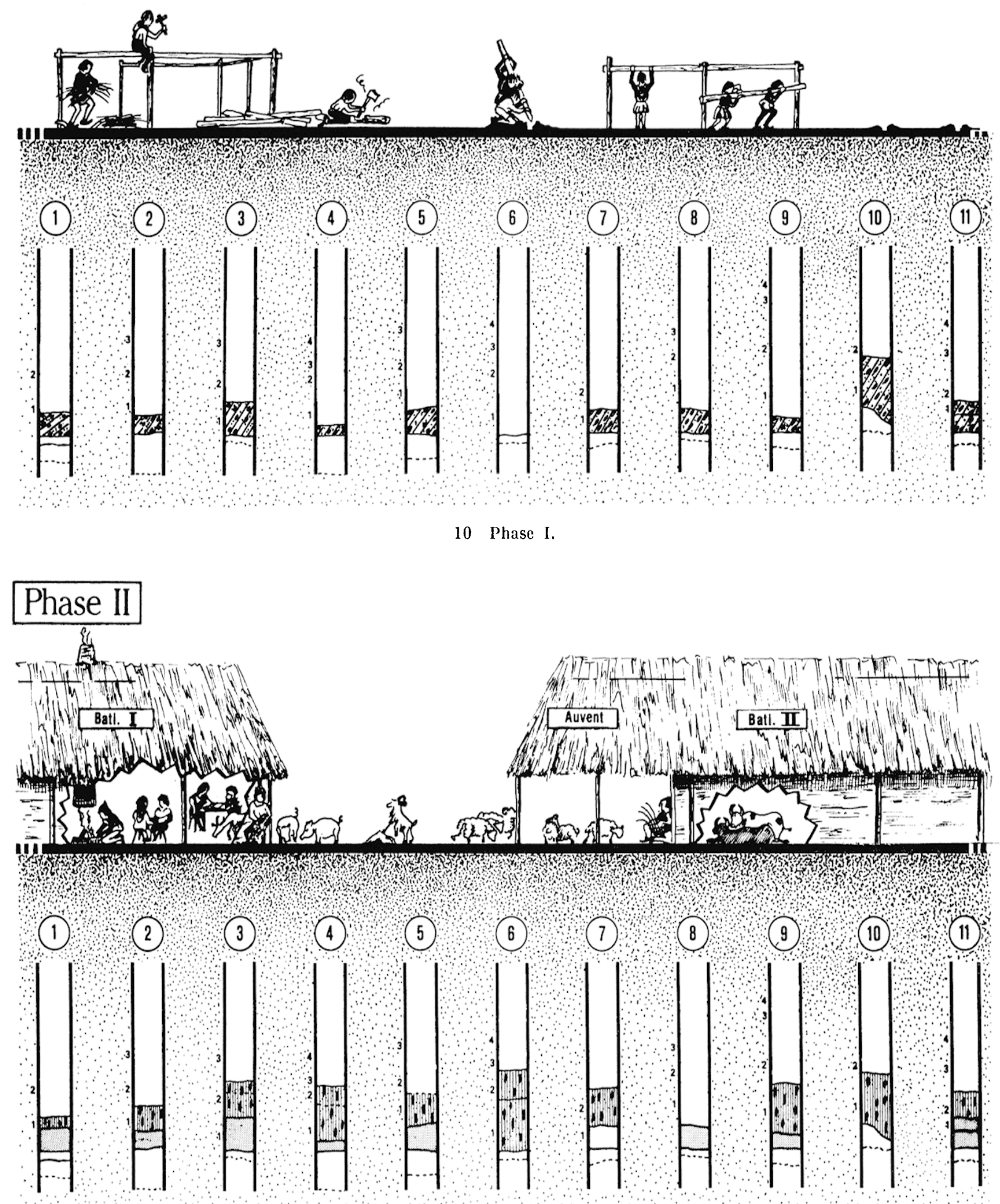

11 Phase II. 


\section{Phase III}
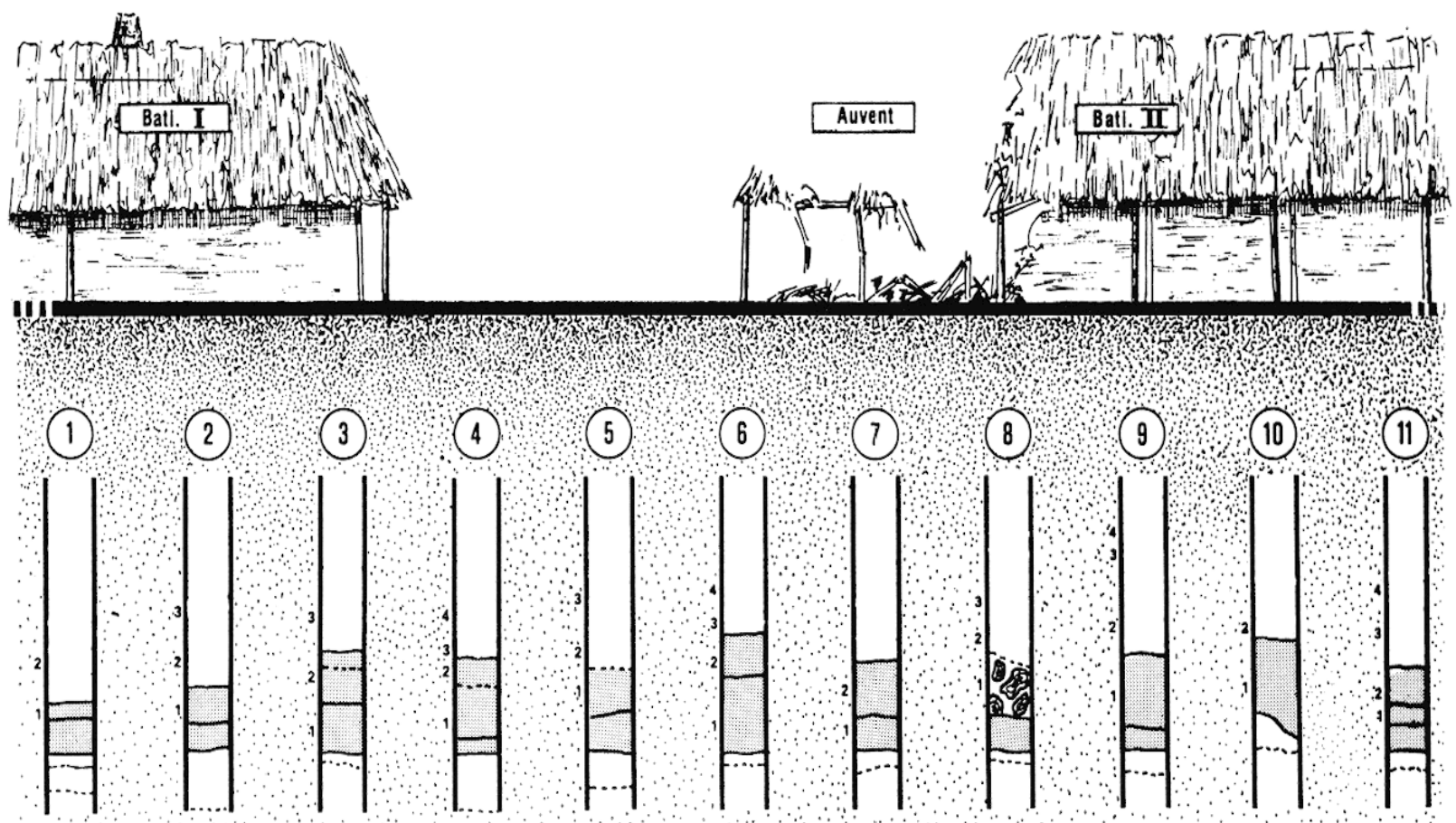

(1) (2)

(3)

(4)

(5)

(6)

(7)

(8)

(0)

(10) (11)
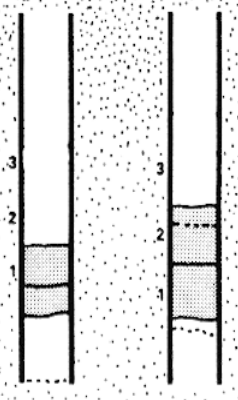

$\left.\right|^{1}$

\begin{tabular}{ll}
\hline \\
2 \\
\hline \\
\hline
\end{tabular}
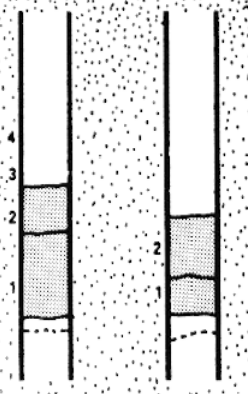

\begin{tabular}{l|l|}
1 \\
3 \\
0
\end{tabular}
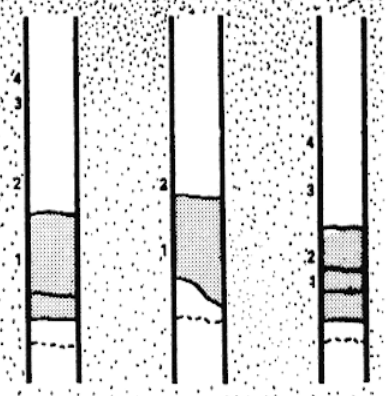

12 Phase III.

\section{PhaseIV}
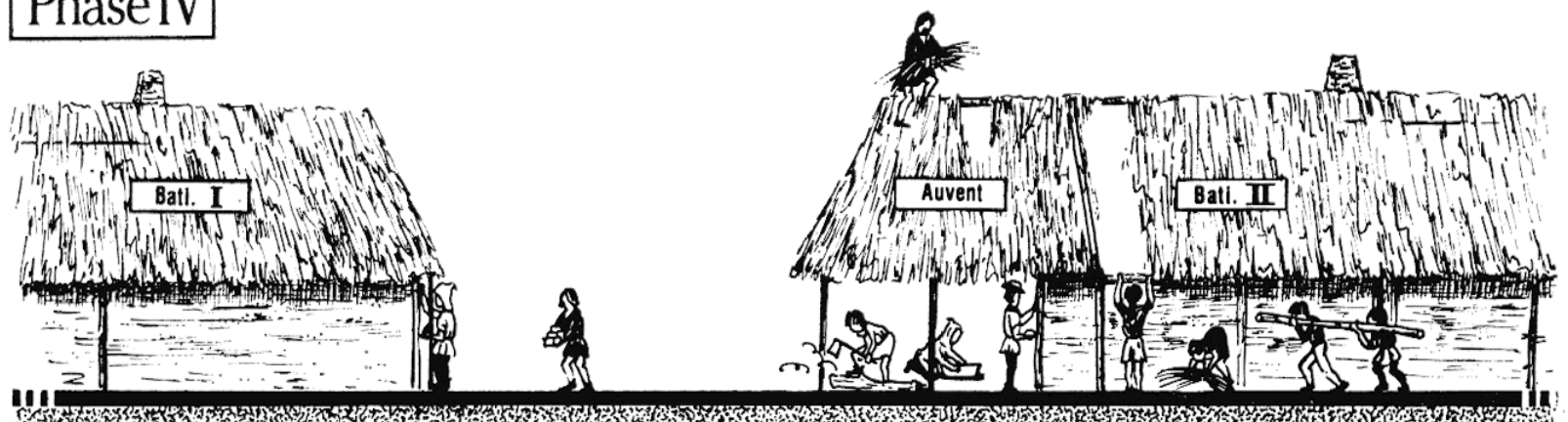

8

(1)

(2)

(3)

(4)

(5)

(6)

(7)

(8)

(9) (10) (11)
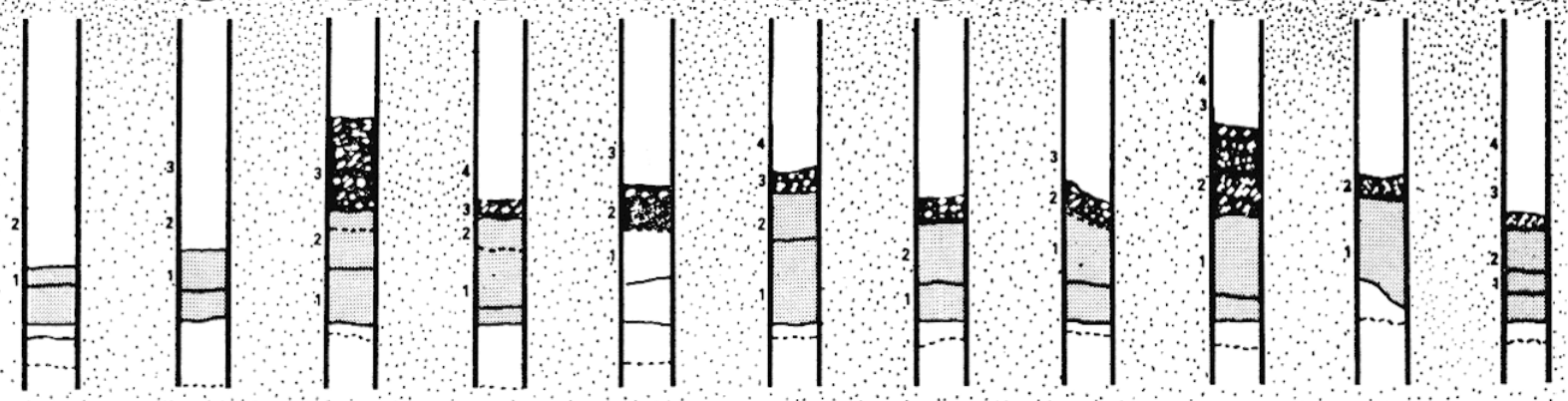

13 Phase IV. 
Le troupeau est parqué dans l'espace compris entre les deux habitations et crée 10 à $20 \mathrm{~cm}$ d'un véritable fumier de stabulation. Vue l'épaisseur de ces niveaux dans les carottes $3,9,10,11$ on peut estimer que les bêtes fréquentaient habituellement les deux habitations, en évitant toutefois la zone foyère du bâtiment I marquée par des dépôts très charbonneux (carottes 1 et 2 , rejets cendreux dans les carottes 4 et 6 ). On peut aussi penser à une zone, entre les deux bâtiments, où aurait été rejeté le fumier des étables. L'épaisseur réelle de ces couches avant leur évolution et leur compaction pouvait être 2 à 4 fois supérieure. On ne note pas, dans cette zone, de fonctionnement de foyer du côté du bâtiment II. Une activité génératrice de phosphates est nettement spécifique au bâtiment I et se fait sentir jusque sous l'auvent du bâtiment II (carotte 7). Cet enrichissement en phosphates pourrait être lié à des rejets et à la décomposition de déchets organiques (autre que les excréments animaux puisque les deux zones ne se recouvrent pas). On ne note pas de traces d'inondations. La carotte 8, par l'absence de sédimentation de fumier et absence, au-delà, de sédiment à fortes teneurs en phosphates, semble bien marquer l'emplacement d'une paroi. Les données de la sédimentologie s'accordent avec un fonctionnement synchrone des deux bâtiments.

\section{PHASE III (fig. 12).}

La carotte 8 qui, précédeminent, n'a enregistré aucun dépôt de fumier présente une couche de gros bois et une structure ouverte. La présence de phosphates pourrait être rattachée à une légère sédimentation de la phase II.

Nous rapportons, en première hypothèse, cette couche à une destruction de la paroi du bâtiment II, accidentelle, ou volontaire, précédant une restructuration, la phase de reconstruction étant repérée dans los sédiment et par la dendrochronologie.

Une autre hypothèse consisterait à rattacher tous ces sédiments à la phase I de construction qui serait ici plus épaisse qu'ailleurs, l'absence de fumier indiquant toujours à cet endroit une non-sédimentation pour la phase II.

La poursuite des fouilles dans le triangle 3-203205 , permettra très certainement de répondre à ces questions.

\section{PHASE IV (fig. 13).}

Ces couches où les débris minéraux sont supérieurs à 20 ou $50 \%$ coupent nettement la séquence stratigraphique en deux parties (fig. 4 a et 5 ). Ces débris minéraux sont composés de grains détritiques, quartz, roches cristallines, calcaires, grès, recoupant l'environnement pétrographique ; s'y ajoutent parfois de nombreux débris d'argile brûlée (carottes $3,4,5)$ et un élément dont nous avons du mal à préciser l'origine (chaux ? tuf chauffé ? (carottes 4, 10, 11). Les pH sont d'une moyenne supérieure à 6,5 (de 7 à 11) et seulement du côté du bâtiment II (fig. $4 \mathrm{~d}$ et 7). Des charbons sont à noter en 6 et 10 . Le synchronisme avec les carottes 1 et 2 n'est pas facile à déceler (il est possible que $1 \mathrm{~S} 1$ et $2 \mathrm{~S} 1$ se soient aussi déposés pendant cette période).

On a là des activités génératrices d'un détritisme minéral. L'origine peut en être une destruction d'éléments construits en terre (au cours d'un très violent orage par exemple), soit au contraire un apport pour construire ou restaurer (torchis des parois, rechappage des foyers). Du côté du bâtiment I (carottes $3,4,5$ ) ce sont des éléments déjà brûlés qui tombent au sol (cheminées ?). On note un apport détritique plus important aux parois $(3-4,8-9)$ : fabrication de torchis ou altération. Les $\mathrm{pH}$ nettement plus élevés dans le bâtiment II pourraient être en rapport avec la présence de "chaux ". Les charbons recueillis carotte 6 indiqueraient la construction et la mise en fonction du foyer du bâtiment II. Les activités diverses continuent et le détritisme végétal reste toujours important, la part minérale ne dépasse jamais $50 \%$.

Cette phase est certainement à rattacher à la deuxième période de construction notée par la dendrochronologie ${ }^{9}$. Cette couche plus minérale devrait permettre de séparer le mobilier provenant de la première période d'occupation (phases I à III) de celle qui va suivre (phase V) qui correspond à une nouvelle organisation des bâtiments. Il s'agit là d'une coupure qu'il est important de noter. Il est plus difficile de juger de sa valeur historique : épisode de la vie quotidienne, changement social, économique? Seules des comparaisons avec d'autres données permettront de répondre à ces questions.

\section{PHASE $V$ (fig. 14).}

Les dépôts sont riches en matière organique et débris végétaux. Ces niveaux présentent de fortes teneurs en "humus" alors que débris végétaux et matière organique atteignent plutôt des valeurs moyennes par rapport aux couches inférieures (fig. $4 \mathrm{c}$ et 5). Les phosphates sont toujours présents du côté du bâtiment 1 (fig. 8). Les agglomérats organiques ne sont plus présents que du côté du bâtiment. I

9 Cf. note 2 . 


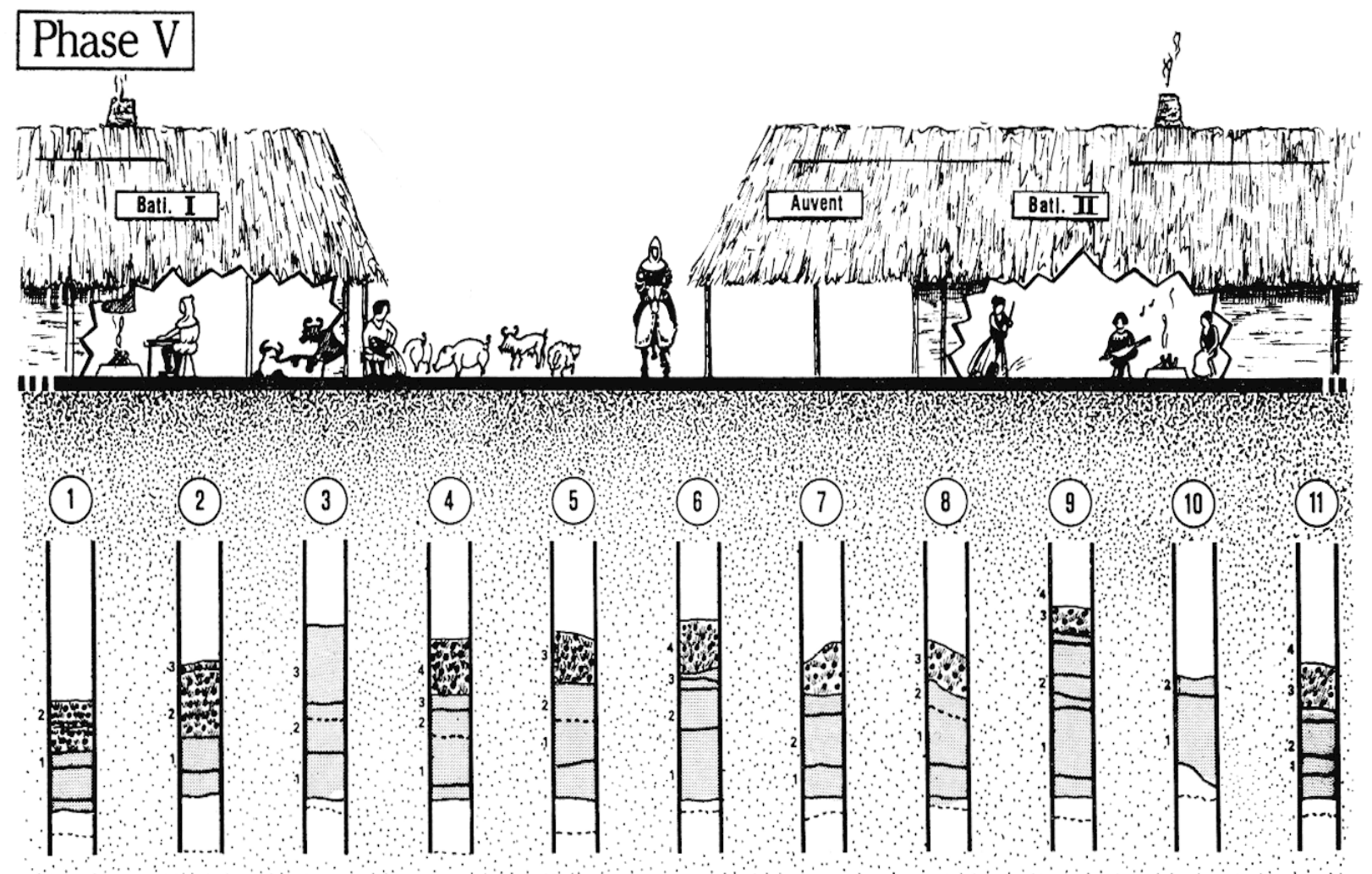

14 Phase V.

PhaseVI

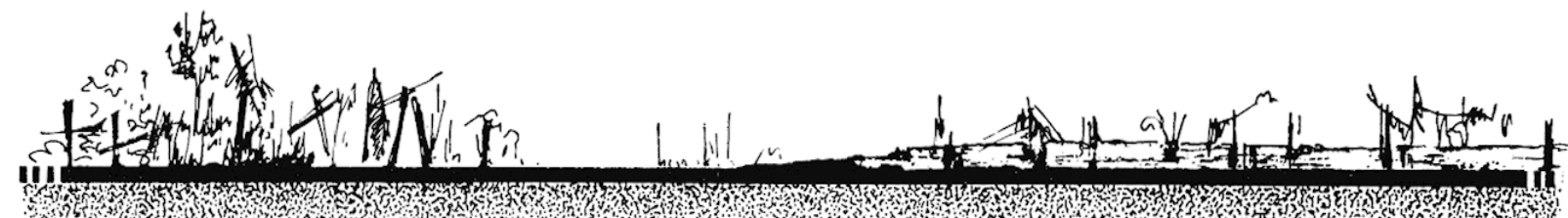

(1) (2)

目
(3)

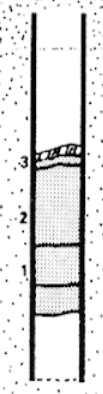

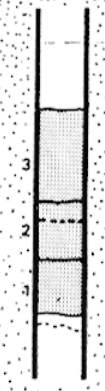

(4)

(5)

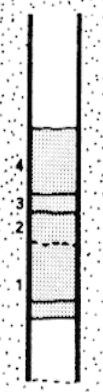

(6)

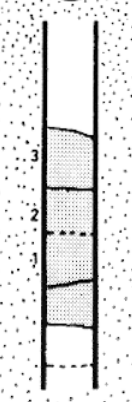

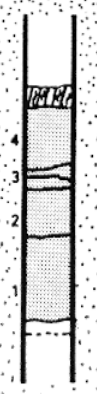

(7)

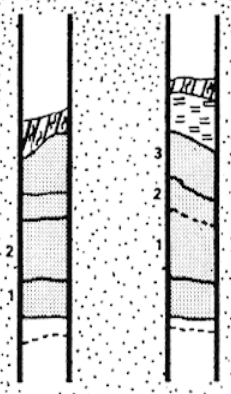

(9)

(10) (11)

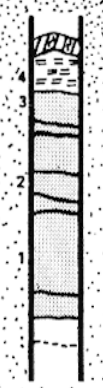

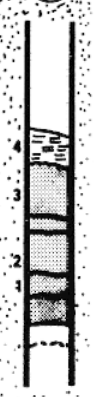

15 Phase VI. 
(fig. 4 b et 6). Les dépôts sont peu développés, surtout au bâtiment II. On ne note aucune trace d'eau.

On assiste à une nouvelle organisation des bâtiments et surtout du bâtiment II. Sa fonction d'étable est totalement abandonnée (son foyer est mis en fonction dès la phase précédente), il s'y dépose peu de chose soit qu'il y ait eu peu d'activités, ou des activités qui n'ont pas été génératrices de sédiment, soit qu'il ait été maintenu propre. Quant au bâtiment I la fonction de stabulation existe encore mais réduite (moins longtemps, moins de bêtes ?); le foyer continue à fonctionner et l'activité génératrice de phosphates subsiste.

\section{PHASE VI (fig. 15).}

On retrouve comme pour la phase I des dépôts végétaux, peu ou non structurés, non compactés (carottes $9 \mathrm{~S} 4,10 \mathrm{~K} 2$, $11 \mathrm{~S} 3$, qui pourraient appartenir à la phase $\mathrm{V}, 2$ au-dessus de S3). Seuls les échantillons 9 S4 et 11 S3 ont été analysés. Les carottes 6 à 9 présentent des formations à lacis de racines de roseaux en position terminale. Une formation crayeuse se trouve en position intermédiaire en carotte 8 et terminale en 11. Ce sont les seules craies supérieures reconnues dans ces carottes.

C'est la phase d'abandon, de destruction. Le matériel se dépose rapidement. Il ne semble pas que l'eau intervienne comme cause de départ, mais cela reste très difficile à prouver. Il n'est pas possible de dire avec quelle rapidité s'est déposée la craie supérieure.

La présence d'"humus", matière organique fine de biodégradation, dans ces sédiments et ceux de la phase précédente, pourrait signifier un abandon et une dégradation du matériel organique ayant duré un certain temps avant son enfouissement sous l'eau et les craies. Des réponses pourront être trouvées dans l'étude de séquences sédimentaires pénécontemporaines du site.

Une partie des sédiments de la phase $\mathrm{V}$ pourrait aussi correspondre à la phase d'abandon. On est de toute façon frappé par le peu de matériel engendré par la destruction des maisons. On doit supposer qu'une grande partie a été déblayée par l'eau lorsqu'elle est arrivée sur le site. Il se peut qu'on n'ait là qu'une partie de la séquence, tronquée par l'érosion.

L'abondance du mobilier archéologique que l'on explique généralement par un départ précipité, ne fournit pas à notre avis, de preuve quant à la rapidité de l'abandon. L'étude stratigraphique, tout au long de laquelle les objets utilitaires, parures, jeux, sont nombreux, montre une sédimentation régulière des phases I à $\mathrm{V}$ au cours des 30,40 années d'occupation. Les raisons de l'abondance du mobilier paraissent devoir être recherchées ailleurs.

Jacques-Léopold Brochier

N.B. Je remercie MM. B. Daxgreaux, P. Mille et M. Colardelle pour leur collaboration sur le terrain, ainsi que MM. M. Joos et F. Scheller pour leur aide au laboratoire de sédimentologie du séminaire de préhistoire de l'Université de Bâle, et M. G.-C. DaIıly pour ses remarques et corrections. Le présent travail a été effectué au Laboratorium für Urgeschichte der Universität, Altere und Naturwissenschafliche Abteilung, Basel, CH.

Le dessin de la figure 1 est de B. Dangreaux, de la figure 2 : J.-L. Brochicr, des figures 4 à 18 : Agnès REvErT (DNAT, Valence). La photographic de la figure 3 est de B. DANGREAU. 